\title{
Untersuchungen über postmortale Verände- rungen des Bestandes niedermolekularer Stickstoffverbindungen in der Muskulatur von Seefischen
}

\author{
Von Heinz Schaefer \\ Aus der Biologischen Anstalt Helgoland
}

(Mit 12 Tabellen)

\begin{abstract}
Es ist häufig versucht worden festzustellen, welche Beziehungen zwischen dem Gehalt an bestimmten Stoffen und dem Verderben des Fischfleisches bestehen. Auf Grund der Bedeutung der stickstoffhaltigen Substanzen wandte sich eine größere Anzahl von Forschern dem Studium der Veränderungen des Bestandes der freien Aminosäuren in der Muskulatur zu. Es zeigte sich dabei, daß sich das Muster der freien Aminosäuren im Verlauf der postmortalen Lagerung des Fischfleisches in charakteristischer Weise verändert. Daraufhin hat es nicht an Versuchen gefehlt, Testverfahren für den Grad der Verderbnis auf Grund der Konzentrationsveränderungen gewisser Aminosäuren und auch Amine auszubauen.

Bisher wurden vorwiegend Fische von großer wirtschaftlicher Bedeutung untersucht, wobei die Verhältnisse beim Kabeljau (Dorsch) das größte Interesse hervorriefen. In den weitaus meisten Fällen handelte es sich um Fische, die nach dem Fang auf übliche Weise gelagert wurden, also in Eis verpackt oder tiefgefroren und dann erst nach der Anlandung zur Analyse kamen. Somit war häufig nicht bekannt, wie lange Zeit die Fische schon tot waren, und auch über den Anfangszustand des Aminosäurenpools im frischgefangenen Fisch wußte man nichts. Außerdem war die Vorbehandlung des Untersuchungsmaterials häufig unterschiedlich: von einer Art wurden Filets, von einer anderen ganze Fische und von einer dritten schließlich ausgenommene Tiere untersucht.

Es erschien wünschenswert, diese Untersuchungen wie folgt zu ergänzen:

1. Untersuchung der postmortalen Veränderungen vom Zeitpunkt des Fanges an.

2. Untersuchung von weiteren Fischarten.
\end{abstract}

Deutscher Beitrag zum Polarfrontprogramm des Internationalen Geophysikalischen Jahres 1958

Der Deutschen Forschungsgemeinschaft danke ich ergebenst für die Gewährung eines Stipendiums und einer Sachbeihilfe. 
3. Untersuchung der aus demselben Fang stammenden Fische nach Lagerung unter verschiedenen Bedingungen.

4. Untersuchung über den Einfluß von Haut und inneren Organen auf die postmortalen Veränderungen in der Muskulatur.

Solche Arbeiten ließen sich nur an Bord eines Schiffes durchführen. Gelegenheit dazu boten die Forschungsfahrten von FFS „Anton Dohrn“ im Internationalen Geophysikalischen Jahr 1958. Das verlangte allerdings gewisse Einschränkungen der Versuche, so vor allem im Hinblick auf die Versuchsdauer und Probeentnahme auf Grund der Witterungsverhältnisse. Die Auswahl der Fische richtete sich nicht nach ihrer kommerziellen Bedeutung, sondern es wurden solche Arten bearbeitet, von denen ein Fang eine genügende Anzahl Tiere einbrachte. Meines Wissens ist über die postmortalen Veränderungen des Aminosäurenbestandes von drei der untersuchten Spezies nichts bekannt, der Rotbarsch war einmal (ein 6 Monate eingefrorenes Filet) Untersuchungsobjekt von Dietrich (1956).

In dieser Arbeit soll über auffälligere Veränderungen des Bestandes niedrigmolekularer Stickstoffverbindungen der Muskulatur auf Grund papierchromatographischer Untersuchungen berichtet werden. Im allgemeinen wird nicht mitgeteilt, wenn sich auf den Papierchromatogrammen der Fleck einer Aminosäure im Verlauf der Verderbnis nur wenig vergrößert oder verkleinert. Die Veränderung der Flecken, also der Konzentration, soll deutlich erkennbar sein. Zu den auffälligen Befunden gehören besonders das Neuauftreten oder aber das gänzliche Verschwinden einer Substanz, was streng genommen bedeutet, daß die betreffende Verbindung entweder jetzt erst nachweisbar geworden oder unter die Nachweisbarkeitsgrenze gelangt ist. Die sich aus den einzelnen Versuchen ergebenden Befunde werden miteinander verglichen, um festzustellen, welche Unterschiede zwischen den Fischarten und zwischen den Lagerungs- und Behandlungsweisen bestehen. Unter Lagerungsart ist hier zu verstehen: tiefgefroren $\left(-22\right.$ bis $\left.-26^{\circ} \mathrm{C}\right)$, Lagerung in Eis (wie es auf Fischdampfern gehandhabt wird, etwa $0^{\circ} \mathrm{C}$ ), Lagerung an Deck bei Außentemperatur $\left(3-12^{\circ} \mathrm{C}\right)$. Behandlungsarten sind hier: ganze Fische (nicht ausgenommen), ausgenommene Fische, Filets mit oder ohne Haut.

\section{Methodik}

Die Methoden wurden in einer früheren Arbeit (Schaefer 1962a) beschrieben. Zur papierchromatographischen Analyse wurden folgende Extraktmengen aufgetragen:

Rotbarsch und Kabeljau: je 0,06 g Frischgewicht,

Wittling, Knurrhahn und Glasauge: je 0,08 $\mathrm{g}$ Frischgewicht,

sowie solche Mengen, die 0,1 mg Kjeldahl-Stickstoff oder 0,04 mg Aminostickstoff entsprachen.

\section{Ergebnisse}

Die Fangplätze sind in Tabelle 1 angegeben.

Das Muster der freien Aminosäuren und der verwandten Verbindungen in der Muskulatur der frischgefangenen Fische dieser Serie wurde in einer 
Tabelle 1: Fangplätze der untersuchten Fische

\begin{tabular}{|c|c|c|c|c|c|c|}
\hline Art & Station & Position & Datum & $\begin{array}{l}\text { Fangtiefe } \\
\text { m }\end{array}$ & $\begin{array}{c}\text { Salzgehalt, } \\
\% \% 0\end{array}$ & Temperatur \\
\hline Kabeljau & 2598 & $\begin{array}{ll}62^{\circ} 30^{\prime} & \mathrm{N} \\
40^{\circ} 30^{\prime} & \mathrm{W}\end{array}$ & 10.9. & $240-270$ & & \\
\hline Knurrhahn & 2233 & $\begin{aligned} 50^{02} 5^{\prime} & \mathrm{N} \\
0^{0} 12^{\prime} & \mathrm{W}\end{aligned}$ & 1.3. & $49-56$ & 35,07 & 8,48 \\
\hline Glasauge & 2301 & $\begin{array}{ll}63^{0} 05^{\prime} & \mathrm{N} \\
23^{0} 57^{\prime} & \mathrm{W}\end{array}$ & 28.3. & $430-460$ & 35,19 & 6,45 \\
\hline Wittling & 2232 & $\begin{array}{c}50^{0} 40,5^{\prime} \mathrm{N} \\
0^{0} 44^{r} \quad \mathrm{O}\end{array}$ & 1.3. & $47-50$ & 34,83 & 7,25 \\
\hline Rotbarsch & 2306 & $\begin{array}{ll}64^{0} 28^{\prime} & \mathrm{N} \\
35^{0} 11^{\prime} & \mathrm{W}\end{array}$ & 4.4. & 405 & & \\
\hline Rotbarsch & 2571 & $\begin{array}{l}65^{0} 18,5^{\prime} \mathrm{N} \\
35^{0} 21^{\prime} \mathrm{W}\end{array}$ & 4. 9. & 300 & 34,84 & 3,54 \\
\hline
\end{tabular}

Soweit gleichzeitig hydrographische Messungen vorgenommen wurden, besorgte mir Herr Prof. Dr. Kalle, Hamburg, liebenswürdigerweise diese Angaben.

früheren Arbeit (SCHAEFer 1962 a) beschrieben. Dort ist ebenfalls ein schematisches Papierchromatogramm mit Angabe der Orte der zitierten unbekannten Substanzen gebracht worden.

In den Tabellen werden folgende Abkürzungen benutzt:

$\begin{array}{llll}\text { Ala } & =\text { Alanin } & \text { Lys } & =\text { Lysin } \\ \text { Arg } & =\text { Arginin } & \text { Met } & =\text { Methionin } \\ \text { Asp } & =\text { Asparaginsäure } & \text { MetHis } & =\text { Methylhistidin } \\ \beta \text {-Ala } & =\beta \text {-Alanin } & \text { Opro } & =\text { Oxyprolin } \\ \alpha-\text { Abs } & =\alpha \text {-Aminobuttersäure } & \text { Orn } & =\text { Ornithin } \\ \beta-\text { Abs } & =\beta \text {-Aminobuttersäure } & \text { Phe } & =\text { Phenylalanin } \\ y \text {-Abs } & =\gamma \text {-Aminobuttersäurc } & \text { Pro } & =\text { Prolin } \\ \text { Citr } & =\text { Citrullin } & \text { Tau } & =\text { Taurin } \\ \text { Cys } & =\text { Cystin/Cystein } & \text { Thr } & =\text { Threonin } \\ \text { Glu } & =\text { Glutaminsäure } & \text { TMO } & =\text { Trimethylaminoxyd } \\ \text { Gly } & =\text { Glykokoll/Serin } & \text { Try } & =\text { Tryptophan } \\ \text { His } & =\text { Histidin } & \text { Tyr } & =\text { Tyrosin } \\ \text { Ileu } & =\text { Isoleucin } & \text { Val } & =\text { Valin } \\ \text { Leu } & =\text { Leucin } & & \end{array}$

Die noch nicht identifizierten Substanzen sind mit Ziffern oder Buchstaben bezeichnet. Histidin konnte nicht immer einwandfrei von Methylhistidin unterschieden werden, "His" kann daher auch mit "MetHis" identisch sein.

Bei besonders starken Veränderungen werden die betreffenden Substanzen in den Tabellen kursiv gedruckt.

Bei den Temperaturangaben der Lagerungsversuche an Deck bandelt es sich um Tagesdurchschnittswerte.

\section{Kabeljau (Gadus morhua L.)}

Über die Veränderungen des Aminosäurenpools in der Kabeljaumuskulatur ist von mehreren Autoren berichtet worden. Bei den meisten Arbeiten handelt es sich um den Dorsch der Nordsee bzw. um angelandete Fische, von denen der Zeitpunkt des Fanges nicht bekannt war.

Es wurden Filets weiblicher Fische untersucht, die in Eis verpackt oder tiefgefroren aufbewahrt waren. 
Die tiefgefrorenen Filets lassen innerhalb von 12 Tagen keine Veränderungen erkennen.

Bei den in Eis verpackten Filets (Tabelle 2) kommt es schon nach 21/2tägiger Lagerung zu einer Zunahme von $\beta$-Alanin und Methylhistidin. Daß es sich dabei um den Abbau von Anserin handelt, ist wahrscheinlich, wenn auch die entsprechende Abnahme von Anserin erst nach weiteren Tagen erkennbar ist. Der Anserinfleck ist auf den Papierchromatogrammen auf Grund

Tabelle 2: Kabeljau

Filets auf Eis

\begin{tabular}{|c|c|c|c|c|}
\hline $\begin{array}{l}\text { Lagerungsdauer } \\
\text { in Tagen }\end{array}$ & Zunahme & Abnahme & Neuauftreten & Verschwinden \\
\hline \multicolumn{5}{|l|}{1} \\
\hline \multirow[t]{2}{*}{$2^{1 / 2}$} & $\beta$-Ala & Arg & & \\
\hline & MetHis & Pro & & \\
\hline \multirow{2}{*}{$\begin{array}{l}5^{1 / 2} \\
9\end{array}$} & & Anserin &, $9^{\prime \prime}$ & \\
\hline & Asp & TMO & & \\
\hline \multirow[t]{4}{*}{12} & Methis & Tau & ${ }_{n} 13^{\prime \prime}$ & \\
\hline & $\beta-A l a$ & Cys & & \\
\hline & Leu &, 5 & & \\
\hline & Ileu & & & \\
\hline
\end{tabular}

schlechter Anfärbbarkeit mit Ninhydrin nicht sehr deutlich, und Konzentrationsveränderungen sind daher schwieriger zu erkennen. Die Zunahme von Leucin und Isoleucin nach 9-12 Tagen ist nicht stark. Sie erreicht nicht das Ausmaß, das bei manchen Fischarten im Stadium der Verderbnis gefunden wird. Doch dürfte das Auftreten von Fleck „13“ (wie später hervorgehoben wird) auf beginnendes Verderben schließen. Bemerkenswert ist das Erscheinen

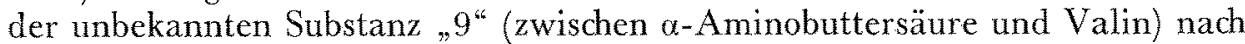
5 Tagen. Diese Verbindung konnte bei anderen Fischarten direkt nach dem Fang nicht mit Sicherheit nachgewiesen werden, wurde aber wohl bei frischgefangenen Nagelrochen (Raja clavata) und mehreren marinen Evertebraten gefunden (Schaefer 1962b). Bei den in dieser Arbeit untersuchten Tieren wurde diese Substanz nur noch bei den in Eis gelagerten Knurrhähnen festgestellt. Nach neuntägiger Lagerung tritt eine weitere unbekannte Verbindung auf (zwischen Prolin und $\alpha$-Aminobuttersäure). Nach 5 Tagen wird das Erscheinen eines rosa-gefärbten Flecks auf der Höhe von Valin, aber außerhalb des Aminosäurenmusters, beobachtet.

\section{Knurrhahn (Trigla cuculus L.)}

Sofort nach dem Fang wurden Knurrhähne von 21-30 cm Länge unausgeweidet in Eis verpackt oder in Eimern auf Deck bei Außentemperatur aufbewahrt. $\mathrm{Zu}$ den in Tabelle 3 angegebenen Zeiten wurden jeweils 3-4 Tiere entnommen und die Muskulatur zu Extrakten verarbeitet.

Bei den bei Außentemperatur gelagerten Fischen sind bereits in den ersten Tagen Veränderungen des Aminosäurenbestandes festzustellen. Zwischen dem 3. und 7. Tag aber nehmen die Konzentrationen der meisten Stickstoffverbindungen durchweg sehr erheblich zu. Trimethylaminoxyd nimmt in dieser Periode stark ab, Phenylalanin, Histamin und Fleck „20" treten auf den 
Tabelle 3: Knurrhahn

\begin{tabular}{|c|c|c|c|c|c|c|c|c|c|}
\hline \multirow{2}{*}{$\begin{array}{l}\text { Lage- } \\
\text { rungs- } \\
\text { dauer } \\
\text { in } \\
\text { Tagen }\end{array}$} & \multicolumn{5}{|c|}{ bei Außentemperatur } & \multicolumn{4}{|c|}{ in Eis } \\
\hline & $\begin{array}{l}\text { Tempe- } \\
\text { ratur }\end{array}$ & $\begin{array}{l}\mathrm{Zu}- \\
\text { nah- } \\
\text { me }\end{array}$ & $\begin{array}{c}\mathrm{Ab}- \\
\text { nah- } \\
\text { me }\end{array}$ & $\begin{array}{l}\text { Neuauf- } \\
\text { treten }\end{array}$ & $\begin{array}{l}\text { Ver- } \\
\text { schwin- } \\
\text { den }\end{array}$ & $\begin{array}{c}\mathrm{Zu}- \\
\text { nahme }\end{array}$ & $\begin{array}{c}\text { Ab- } \\
\text { nahme }\end{array}$ & $\begin{array}{c}\text { Neuauf- } \\
\text { treten }\end{array}$ & $\begin{array}{l}\text { Ver- } \\
\text { schwin- } \\
\text { den }\end{array}$ \\
\hline 1 & & $\begin{array}{l}\text { Giy } \\
\text { Glu }\end{array}$ & & $\mathrm{T}_{y \mathrm{r}}$ & & & & & \\
\hline & & Pro & & & & & & & \\
\hline 2 & $10-12^{0}$ & Arg & & $\beta$-Ala & & & & & \\
\hline 3 & $11^{0}$ & $\mathrm{Val}$ & &, $13 "$ & & & & & \\
\hline \multirow[t]{17}{*}{7} & & Gly & TMO & Phe & & & & & \\
\hline & & Glu & & Histamin & & & & & \\
\hline & & Asp & & & & & & & \\
\hline & & His & & & & & & & \\
\hline & & $\begin{array}{l}\text { Ala } \\
B \text {-Ala }\end{array}$ & & & & & & & \\
\hline & & Opro & & & & & & & \\
\hline & & Thr & & & & & & & \\
\hline & & Tyr & & & & & & & \\
\hline & & $\mathrm{Val}$ & & & & & & & \\
\hline & & $a-A b s$ & & & & & & & \\
\hline & & $\gamma-A b s$ & & & & & & & \\
\hline & & Leu & & & & & & & \\
\hline & & Ileu & & & & & & & \\
\hline & & Citr & & & & & & & \\
\hline & & $" 6^{4}$ & & & & & & & \\
\hline & & "11" & & & & & & & \\
\hline & & $.13 “$ & & & & & & & \\
\hline 9 & & & & & & Citr & & 134 & \\
\hline 13 & & & & & & Val & & $9^{\prime \prime}$ & Anserin \\
\hline \multirow[t]{7}{*}{20} & & & & & & Asp & Lys & Phe & Orn \\
\hline & & & & & & Tyr & Arg & & TMO \\
\hline & & & & & & $\mathrm{Val}$ & Tau & & \\
\hline & & & & & & $a-A b s$ & Pro & & \\
\hline & & & & & & Leu &, 5 & & \\
\hline & & & & & & Ileu & & & \\
\hline & & & & & & $" z^{\prime \prime}$ & & & \\
\hline 23 & & & & & & Phe & Pro & Histamin & \\
\hline & & & & & & Leu &, $5^{\prime \prime}$ & & \\
\hline & & & & & & Ileu & & & \\
\hline & & & & & & Citr & & & \\
\hline
\end{tabular}

Papierchromatogrammen neu auf. Gleichzeitig macht sich sehr starker Verderbnisgeruch bemerkbar, die Fischhaut ist stark angegriffen.

Die in Eis verpackten Fische zeigen ein wesentlich anderes Bild. Erst nach 9-13 Tagen wird die Zunahme einiger weniger Aminosäuren bei gleichzeitigem Verschwinden des Peptids Anserin beobachtet. Zwischen dem 13. und 20. Tag sowie an den darauffolgenden Tagen treten stärkere Veränderungen auf, die aber bei weitem nicht die Intensität haben wie die bei Außentemperatur gelagerten Fische. Neben der Zunahme der Mengen verschiedener Verbindungen ist eine Abnahme anderer Substanzen zu erkennen. Das Erscheinen von Fleck "20" ist nicht feststellbar, dagegen tritt hier, aber nicht bei den in Eis gelagerten Fischen, der Fleck "9" auf. 
Zwischen den Lagerungsbedingungen gibt es also vier auffällige Unterschiede: 1. die zeitliche Verschiebung der Vorgänge, 2. die verschieden große Intensität der Vorgänge, 3. stärkere Abnahme von Konzentrationen nur bei Eislagerung, 4. die Veränderungen betreffen zum Teil unterschiedliche Substanzen.

\section{Glasauge (Argentina silus Ascanius)}

Sofort nach dem Fang wurde eine größere Anzahl von annähernd gleich großen Fischen in einen Eimer gelegt und bei Außentemperatur aufbewahrt. Fast täglich wurden 2-3 Fische für die Analyse entnommen. Eine Anzahl weiterer Tiere aus demselben Fang wurde entweder sofort tiefgefroren oder als nicht ausgenommene Fische oder als Filets mit Haut in Eis verpackt und zu den angegebenen Zeiten analysiert.

Bei den tiefgefrorenen Fischen sind im Verlauf einer 22tägigen Lagerung papierchromatographisch keine nennenswerten Veränderungen zu sehen.

Tabelle 4: Glasauge

Ganze Fische bei Außentemperatur gelagert

\begin{tabular}{|c|c|c|c|c|c|}
\hline $\begin{array}{l}\text { Lagerungsdauer } \\
\text { in Tagen }\end{array}$ & Temperatur & Zunahme & Abnahme & Neuauftreten & Verschwinden \\
\hline 1 & $6^{0}$ & & & & \\
\hline 3 & $6^{\mathbf{a}}$ & & & & \\
\hline 5 & $7^{0}$ & & & & \\
\hline 6 & $5^{0}$ & $" 6^{4}$ & TMO & $\begin{array}{l}\text { "13" } \\
\text { Orn }\end{array}$ & \\
\hline \multirow[t]{7}{*}{7} & $3^{0}$ & Glu & & Phe & \\
\hline & & Asp & & $z^{\prime \prime}$ & \\
\hline & & His & & & \\
\hline & & Pro & & & \\
\hline & & Leu & & & \\
\hline & & Ileu & & & \\
\hline & & $" 13 "$ & & & \\
\hline \multirow[t]{6}{*}{8} & $5^{0}$ & Arg & TMO & $" 20^{\prime \prime}$ & \\
\hline & & Ala & & $" 2^{\prime \prime}$ & \\
\hline & & $\mathrm{Val}$ & & & \\
\hline & & $\alpha-A b s$ & & & \\
\hline & & $\gamma$-Abs & & & \\
\hline & & $\begin{array}{l}\text { Leu } \\
\text { Ileu }\end{array}$ & & & \\
\hline \multirow[t]{3}{*}{9} & $7^{0}$ & Thr & & & TMO \\
\hline & &, $\mathrm{z}^{\prime \prime}$ & & & \\
\hline & & "13" & & & \\
\hline \multirow[t]{2}{*}{10} & $6^{0}$ & Asp & & & \\
\hline & & Phe & & & \\
\hline
\end{tabular}

Die bei Außentemperatur lagernden Fische weisen frühzeitig Verderbniserscheinungen auf (Tabelle 4). Trimethylaminoxyd beginnt nach 6 Tagen sichtbar abzunehmen, Fleck „13“ und Ornithin treten auf. Die größten Veränderungen sind in den beiden nächsten Tagen zu erkennen, die vor allem durch das Erscheinen der bisher auf den Papierchromatogrammen nicht nachweisbaren Substanzen Phenylalanin, Fleck ,20" und , $\mathrm{z}^{\text {" }}$ und durch starkes Ansteigen der Konzentrationen vieler Aminosäuren, vor allem von Leucin, Isoleucin und $\alpha$-Aminobuttersäure gekennzeichnet sind. Nach dem 9. und 10 . 
Tabelle 5: Glasauge

Lagerung in Eis

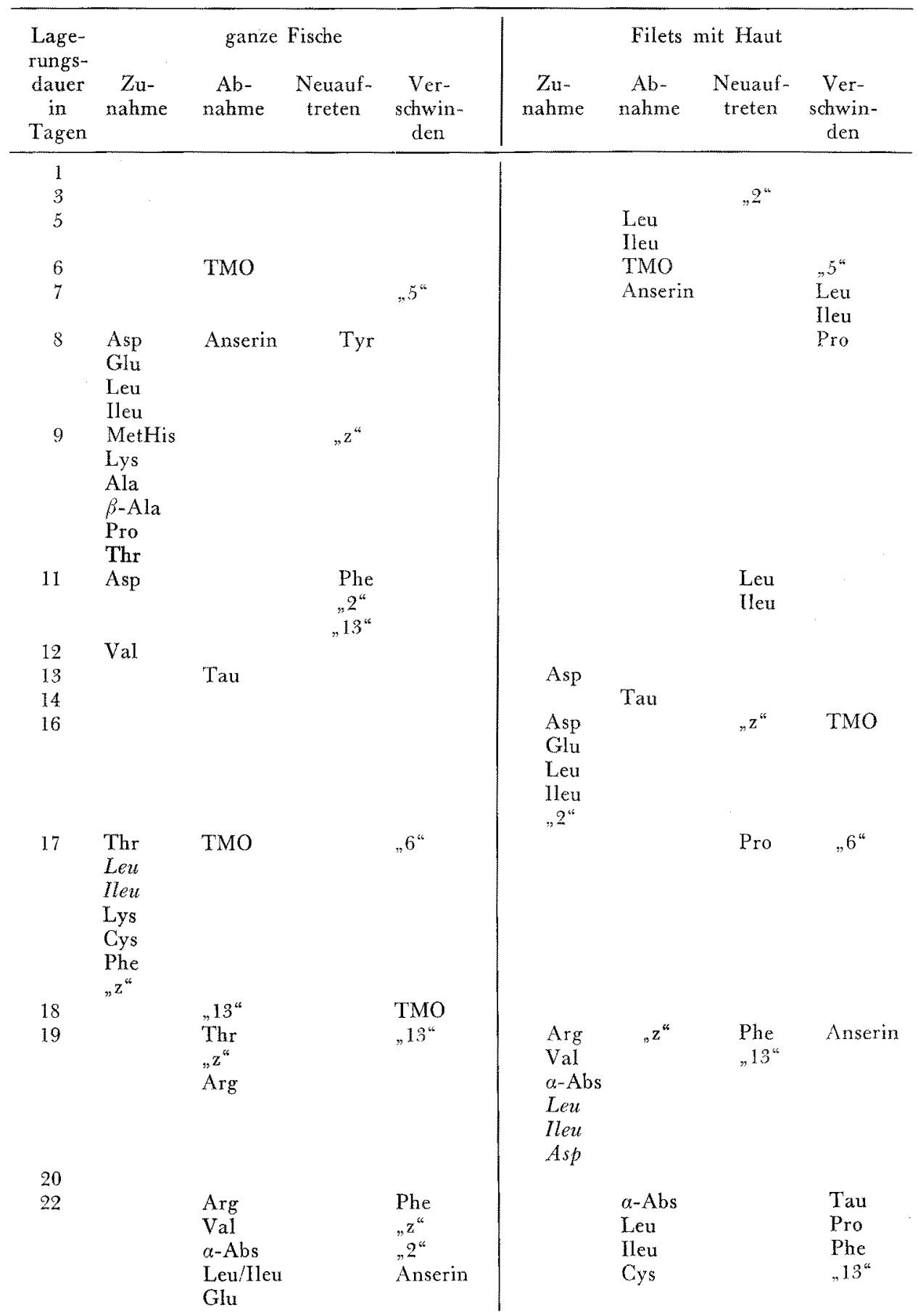

Tag erfolgt weitere Zunahme der Mengen einiger Substanzen, besonders von Asparaginsäure. Die Haut ist nunmehr sehr deformiert, wegen zu starken Geruchs mußte der Versuch abgebrochen werden. 
Bei den in Eis gelagerten Fischen beginnen diese Prozesse etwas später und auch nicht so gleichzeitig wie bei den bei Außentemperatur gelagerten Tieren (Tabelle 5). Nach 8 bis 12 Tagen nimmt bei den nicht ausgenommenen Fischen eine Reihe von Aminosäuren an Menge zu. Einige Substanzen erscheinen zum erstenmal auf dem Papierchromatogramm, Trimethylaminoxyd und Anserin nehmen ab. In der Folge nehmen die Konzentrationen mehrerer Verbindungen weiterhin zu, es beginnt aber auch bei anderen Aminosäuren bereits eine Mengenverringerung bzw. ein völliges Verschwinden. Zuletzt werden nur noch Abbauerscheinungen beobachtet.

Bei den Filets ist bereits nach 5 bis 6 Tagen ein Nachlassen der Mengen mehrerer Substanzen und sogar Verschwinden von Flecken von den Chromatogrammen feststellbar. Eine deutliche Zunahme erfolgt aber erst nach $13 \mathrm{Ta}$ gen. Die letzte Phase entspricht in etwa der der ganzen Fische.

Zwischen nicht ausgenommenen Fischen und Filets sind also bei Eislagerung deutliche Unterschiede zu finden. Abnahme der Konzentrationen und Verschwinden von Substanzen aus dem Nachweisbarkeitsbereich sind bei den Filets früher und stärker festzustellen. Die Mengen verschiedener Verbindungen nehmen bei den Filets jedoch zu einem späteren Zeitpunkt zu, bei ihnen treten auch Phenylalanin, „13“ und „z“ erst später auf. Ein großer Teil der Prozesse wird bei den Filets also erst zu einem späteren Zeitpunkt beobachtet. Merkwürdig erscheint das frühzeitige Verschwinden von Leucin und Isoleucin und Prolin und ihr späteres Wiederauftreten bei den Filets. Die nach 22 Tagen ablaufenden Vorgänge sind bei ganzen Fischen und bei Filets aber ziemlich gleich. Die Mengen einiger Aminosäuren verhalten sich bei beiden Behandlungsarten unterschiedlich, so ist der Asparaginsäurefleck bei den Filets stärker als bei den ganzen Fischen.

Zwischen Lagerung in Eis und Lagerung bei Außentemperatur sind die Unterschiede noch stärker. Bei Außentemperatur verlaufen die Prozesse wesentlich konzentrierter und schneller, aber auch andersartig. Bemerkenswerte Abnahmen von Konzentrationen sind nur bei Trimethylaminoxyd zu beobachten. In der Versuchszeit wird dort auch nur das Verschwinden von Trimethylaminoxyd beobachtet, Fleck "29" tritt nur bei Deckslagerung auf.

\section{Wittling (Merlangius merlangus L.)}

Frischgefangene Wittlinge von $28-40 \mathrm{~cm}$ Länge wurden sowohl bei Außentemperatur als auch in Eis verpackt aufbewahrt. Es wurden jeweils ausgenommene und nicht ausgenommene Fische untersucht.

Die ersten Veränderungen des Aminosäurenbestandes bei Aufbewahrung bei Außentemperatur sind bereits nach 2 Tagen erkennbar (Tabelle 6). Jedoch setzen die weiteren Veränderungen nach 3 bis 4 Tagen bei den nicht ausgenommenen Tieren kompakter ein, während sie sich bei den ausgeweideten Fischen über einen größeren Zeitraum hinziehen. Die Konzentrationen einiger Verbindungen, deren Auftreten bzw. hohe Konzentration mit Verdèrbniserscheinungen gekoppelt sein dürften, nehmen bei den ganzen Fischen früher $z u, z$. B. Leucin/Isoleucin, Phenylalanin, jedoch erscheinen Histamin und die Flecken "13" und „20" bei beiden Behandlungsarten zu denselben Zeitpunkten. Ebenso ist eine Abnahme oder das Verschwinden von Trimethylaminoxyd bei beiden Behandlungsarten zu demselben Termin zu beobachten. Die 
Konzentrationen einiger Aminosäuren verhalten sich bei ausgenommenen und nicht ausgenommenen Fischen sehr unterschiedlich.

Die Unterschiede zwischen ausgenommenen und nicht ausgenommenen Wittlingen sind bei Eislagerung sehr gering (Tabelle 7). Bei den nicht ausgeweideten Fischen erscheint Tryptophan etwas früher auf den Papierchromatogrammen. Es hat den Anschein, als wären die Prozesse bei den nicht ausgenommenen Tieren etwas beschleunigter.

Tabelle 6: Wittling

Aubewahrung bei Außentemperatur

\begin{tabular}{|c|c|c|c|c|c|c|c|c|c|}
\hline \multirow{2}{*}{\multicolumn{2}{|c|}{$\begin{array}{l}\text { Lage- } \\
\text { rungs- } \\
\text { dauer Temp. } \\
\text { in } \\
\text { Tagen }\end{array}$}} & \multicolumn{4}{|c|}{ nicht ausgenommen } & \multicolumn{4}{|c|}{ ausgenommen } \\
\hline & & $\begin{array}{c}\mathrm{Zu}- \\
\text { nahme }\end{array}$ & $\begin{array}{c}\mathrm{Ab}- \\
\text { nahme }\end{array}$ & $\begin{array}{l}\text { Neuauf } \\
\text { treten }\end{array}$ & $\begin{array}{l}\text { Ver- } \\
\text { schwin- } \\
\text { den }\end{array}$ & $\begin{array}{c}Z \mathrm{u}^{-} \\
\text {nahme }\end{array}$ & $\begin{array}{c}A b- \\
\text { nabme }\end{array}$ & $\begin{array}{c}\text { Neuauf- } \\
\text { treten }\end{array}$ & $\begin{array}{l}\text { Ver- } \\
\text { schwin- } \\
\text { den }\end{array}$ \\
\hline \multicolumn{10}{|c|}{ 1. $10-12^{\circ}$} \\
\hline 2 & $11^{0}$ & $\beta$-Ala & & Phe & & & & & \\
\hline \multirow[t]{8}{*}{4} & $8^{0}$ & Glu & Arg & $m 13^{\prime \prime}$ & & $a-A b s$ & TMO & $13^{\prime \prime}$ & Arg \\
\hline & & MetHis & TMO &, $20 "$ & & $\gamma-\mathrm{Abs}$ & &, $20^{\circ}$ & \\
\hline & & & & & & $\beta-A l a$ & & & \\
\hline & & & & & & & & & \\
\hline & & Leu & & & & & & & \\
\hline & & llew & & & & & & & \\
\hline & & $\alpha-\mathrm{Abs}$ & & & & & & & \\
\hline & & $z^{4}$ & & & & & & & \\
\hline \multirow[t]{4}{*}{6} & $7^{0}$ & Leu & & Try & TMO & Pro & & & TMO \\
\hline & & Ileu & &, $11^{\prime \prime}$ & & Val & & & \\
\hline & & Ala & & & &, $20^{\circ}$ & & & \\
\hline & & $\beta$-Ala & & & & $" z^{\prime \prime}$ & & & \\
\hline \multirow{3}{*}{8} & $5^{0}$ & $" 200$ & & & & Leu & & Try & \\
\hline & & & & & & Ileu & & Phe & \\
\hline & & & & & & "13“ & & & \\
\hline \multirow[t]{6}{*}{12} & $5^{0}$ & Cys & Arg & Histamin & n Opro & His & Tau & Met & Opro \\
\hline & & His & Tau & & & Glu & $.5^{\circ}$ &, $11 \%$ & \\
\hline & & $\gamma$-Abs &, $5^{\prime \prime}$ & & & Val & .6 & Histamin & \\
\hline & & & $" 6^{\prime \prime}$ & & & $\alpha-\mathrm{Abs}$ & & & \\
\hline & & & & & & $\gamma$-Abs & & & \\
\hline & & & & & & llen & & & \\
\hline
\end{tabular}

Erwartungsgemäß beginnen die postmortalen Veränderungen des Pools der Stickstoffverbindungen bei den bei Außentemperatur aufbewahrten Wittlingen früher als bei Eislagerung. Bei den ersteren verlaufen die Prozesse bei weitem stärker, und die Veränderungen betreffen eine größere Zahl von Substanzen. Bei Deckslagerung wird aber das Auftreten von Substanz , $7^{\prime \prime}$ und das Verschwinden von Anserin nicht beobachtet, hingegen treten Methionin und Histamin bei den ausgenommenen Tieren bei dieser Lagerungsart auf, nicht aber (oder noch nicht?) auf Eis. 
Tabelle 7: Wittling

Aufbewahrung in Eis

\begin{tabular}{|c|c|c|c|c|c|c|c|c|}
\hline \multirow{2}{*}{$\begin{array}{l}\text { Lage- } \\
\text { rungs- } \\
\text { dauer } \\
\text { in } \\
\text { Tagen }\end{array}$} & \multicolumn{4}{|c|}{ nicht ausgenommen } & \multicolumn{4}{|c|}{ ausgenommen } \\
\hline & $\begin{array}{c}\mathrm{Zu-} \\
\text { nahme }\end{array}$ & $\begin{array}{c}A b- \\
\text { nahme }\end{array}$ & $\begin{array}{c}\text { Neuauf- } \\
\text { treten }\end{array}$ & $\begin{array}{c}\text { Ver- } \\
\text { schwin- } \\
\text { den }\end{array}$ & $\begin{array}{c}\mathrm{Zu}- \\
\text { nahme }\end{array}$ & $\underset{\text { nahme }}{\mathrm{Ab}-}$ & $\begin{array}{c}\text { Neuauf- } \\
\text { treten }\end{array}$ & $\begin{array}{l}\text { Ver- } \\
\text { sdiwin- } \\
\text { den }\end{array}$ \\
\hline 4 & $\begin{array}{l}\text { MetHis } \\
\beta \text {-Ala }\end{array}$ & & Try & & $\begin{array}{l}\text { MetHis } \\
\beta \text {-Ala }\end{array}$ & Cys & & \\
\hline $\begin{array}{l}6 \\
8\end{array}$ & $\beta-A l a$ & Cys & Phe & & & & $\begin{array}{l}\text { Try } \\
\text { Phe }\end{array}$ & \\
\hline 12 & Cys & & $" 13^{\alpha}$ & & $\begin{array}{l}\text { Cys } \\
\beta \text {-Ala }\end{array}$ & TMO & $" 18^{\prime \prime}$ & \\
\hline 16 & & $\begin{array}{l}\text { TMO } \\
\text { Tau }\end{array}$ & $" 7^{16}$ & & & Anserin & $" 7^{\prime \prime}$ & \\
\hline 19 & $\begin{array}{l}\text { Leu } \\
\text { Ileu } \\
\text { Phe } \\
\text { Glu }\end{array}$ & $\begin{array}{c}\text { Anserin } \\
\beta \text {-Ala } \\
\ldots \delta^{\prime \prime}\end{array}$ & & TMO & Glu & $\begin{array}{l}\text { Tau } \\
\text { "5" }\end{array}$ & & TMO \\
\hline 22 & $\begin{array}{l}\text { Asp } \\
\text { Pro } \\
\text { Phe } \\
\text { Val } \\
a-A b s\end{array}$ & & & $\begin{array}{l}\text { Anserin } \\
\text { Orn }\end{array}$ & $\begin{array}{l}\text { Asp } \\
\text { Pro } \\
\text { Phe } \\
\text { Val } \\
a-A b s \\
\gamma \text {-Abs } \\
\text { Leu } \\
\text { Ileu }\end{array}$ & $\begin{array}{l}\text { Anserin } \\
\beta \text {-Ala }\end{array}$ & & $\begin{array}{l}\text { Orn } \\
5^{\prime \prime}\end{array}$ \\
\hline
\end{tabular}

Rotbarsch (Sebastes marinus L.)

Nachdem in früheren papierchromatographischen Untersuchungen (SCHAEFER 1959) keine nennenswerten Veränderungen im Pool der freien Aminosäuren der Muskulatur des Rotbarsches bis zu 12 Stunden nach dem Tod gefunden wurden, unternahm ich neue Versuche, die sich über einen längeren Zeitraum hinstreckten. Es wurden Winter- und Sommerfänge analysiert, um jahreszeitlich bedingte Unterschiede feststellen zu können. Bei den Winteruntersuchungen sollte gleichzeitig der Einfluß der Haut und der inneren Organe überprüft werden.

\section{Winterfang}

Männliche, ca. $55 \mathrm{~cm}$ lange Rotbarsche aus demselben Fang wurden als ganze oder ausgenommene Fische oder als enthäutete Filets in Eis verpackt. Andere Fische desselben Fanges wurden sofort bei -22 bis $-26^{\circ} \mathrm{C}$ tiefgefroren. Nach den angegebenen Lagerungszeiten wurden je 2 Filets bzw. 1 Fisch verarbeitet.

Die Papierchromatogramme der tiefgefrorenen Filets lassen innerhalb von 15 Tagen keine nennenswerten Veränderungen erkennen.

In demselben Zeitraum können bei ganzen und ausgenommenen Rotbarschen, die in Eis verpackt waren, nur geringe Schwankungen beobachtet werden (Tabelle 8). Bei den ausgenommenen Tieren ist der Leucin-IsoleucinKomplex nach 7 Tagen nicht mehr nachweisbar, tritt aber nach 11 Tagen wieder in ungefähr der Initialmenge auf. Nach 8 Tagen wird eine Abnahme 
Tabelle 8: Rotbarsch, Winterfang Lagerung auf Eis

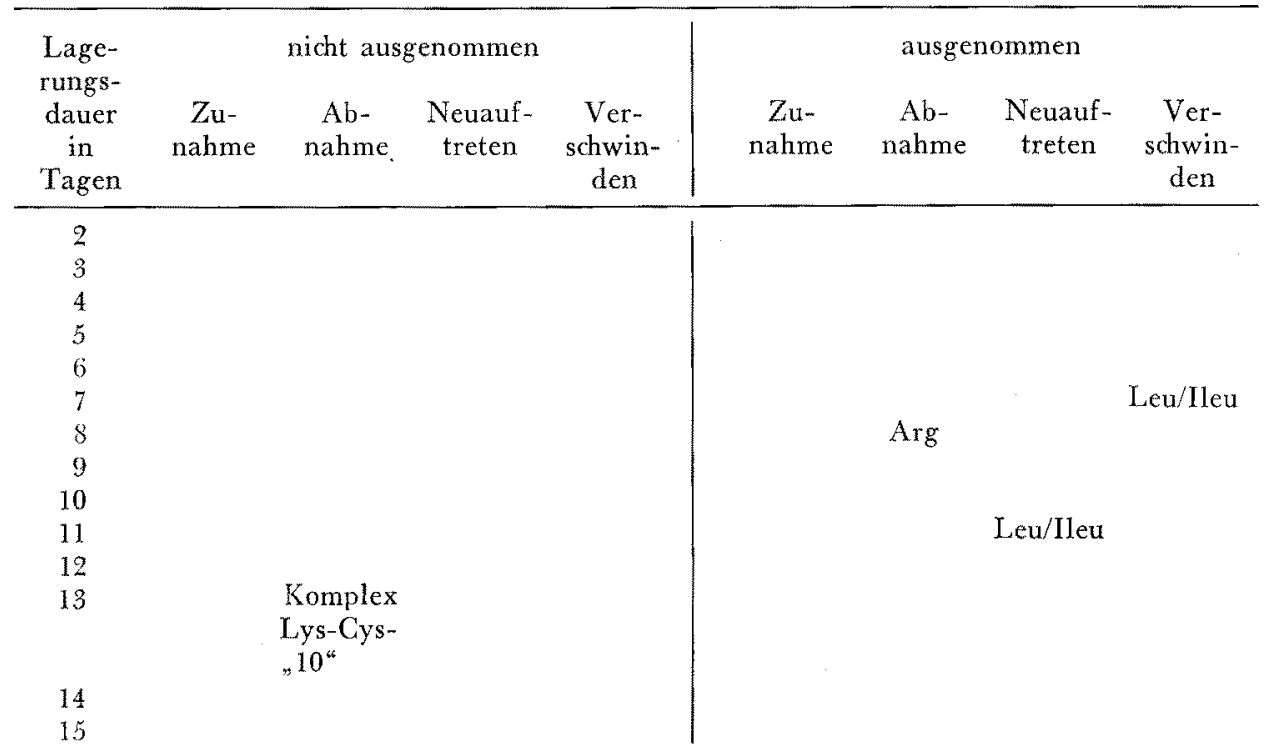

von Arginin beobachtet. Die ganzen Fische zeigen nach 13 Tagen abfallende Mengen des Komplexes Lysin-Cystin-, 10“.

Die in Eis gelagerten Filets bringen jedoch erheblich andere Befunde (Tabelle 9). Das Verschwinden von Leucin/Isoleucin und die Abnahme von Arginin wird schon nach kürzerer Lagerungszeit gefunden, Leucin/Isoleucin treten später wieder auf. Im Verlaufe der Lagerung werden Konzentrationsveränderungen, zunächst besonders Abnahme, auch von etlichen weiteren Substanzen beobachtet. $\beta$-Aminobuttersäure, Arginin und Fleck „10“ verschwinden aus dem Nachweisbarkeitsbereich. Das Auftreten der noch nicht indentifi-

Tabelle 9: Rotbarsch, Winterfang in Eis gelagerte Filets

\begin{tabular}{|c|c|c|c|c|}
\hline $\begin{array}{l}\text { Lagerungsdauer } \\
\text { in Tagen }\end{array}$ & Zunahme & Abnahme & Neuauftreten & Verschwinden \\
\hline \multicolumn{5}{|l|}{1} \\
\hline \multicolumn{5}{|r|}{ Leu/lleu } \\
\hline \multicolumn{5}{|l|}{3} \\
\hline \multirow[t]{2}{*}{4} & & Arg & & \\
\hline & &, $10^{\prime \prime}$ & & \\
\hline \multicolumn{5}{|c|}{ Thr } \\
\hline \multicolumn{5}{|l|}{6} \\
\hline \multirow[t]{2}{*}{8} & & Arg & $2^{\prime \prime}$ & $\beta$-Abs \\
\hline & & Asp & & \\
\hline \multicolumn{5}{|c|}{ TMO } \\
\hline \multicolumn{5}{|l|}{10} \\
\hline \multirow[t]{2}{*}{11} & Asp & & & \\
\hline & Citr & & & \\
\hline 12 & & & $.13^{\prime \prime}$ & \\
\hline 13 & „2“ & TMO & Leu/Ileu &, $10^{\star}$ \\
\hline 14 & $" 13 "$ & & Tyr & Arg. \\
\hline 15 & $" 6^{4}$ & & & \\
\hline
\end{tabular}


zierten Substanzen „2“ und „13“ wird nur bei Filets beobachtet; bei diesen ist eine Veränderung des Komplexes Lysin-Cystin- „10“ insoweit zu erkennen, als Fleck "10" nach 4 Tagen bereits deutlich abnimmt und später verschwindet.

\section{Sommerfang}

Ein wesentlich anderes Bild ergeben die postmortalen Veränderungen in den eisgelagerten Filets des Sommerfanges (Tabelle 10). Nach 6 Tagen wird eine Zunahme von Lysin festgestellt. Nach 14 Tagen werden die Veränderun-

Tabelle 10: Rotbarsch, Sommerfang in Eis gelagerte Filets

\begin{tabular}{|c|c|c|c|c|}
\hline $\begin{array}{l}\text { Lagerungsdauer } \\
\text { in Tagen }\end{array}$ & Zunahme & Abnahme & Nevauftreten & Verschwinden \\
\hline \multicolumn{5}{|l|}{$1^{1 / 2}$} \\
\hline \multicolumn{5}{|l|}{3} \\
\hline \multicolumn{5}{|c|}{ Pro } \\
\hline \multirow[t]{2}{*}{6} & Lys & & Tyr & \\
\hline & & & $" 13 "$ & \\
\hline \multirow[t]{3}{*}{11} & & TMO & $n^{2 "}$ & \\
\hline & & Arg & & \\
\hline & & $" 10^{\prime \prime}$ & & \\
\hline \multirow{8}{*}{14} & Glu & Cys & Met & Opro \\
\hline & $A s p$ & Arg & Phe & \\
\hline & Tyr & Tau & "z" & \\
\hline & Val & TMO & & \\
\hline & $\alpha-\mathrm{Abs}$ & $" 10^{\prime \prime}$ & & \\
\hline & Lett & & & \\
\hline & Ileu & & & \\
\hline & „13" & & & \\
\hline \multirow[t]{3}{*}{18} & Tyr & Cys & & TMO \\
\hline & Met & & & $" 10^{\prime \prime}$ \\
\hline & Phe & & & $" 13^{\prime \prime}$ \\
\hline
\end{tabular}

gen sehr deutlich, die Konzentrationen mehrerer Aminosäuren nehmen ungleich stärker zu als bei den Winterfilets. Das gilt besonders für die in der Tabelle kursiv gedruckten Verbindungen. Zwischen dem 11. und 14. Lagerungstag tritt also eine kritische Phase auf, die bei den Winterfischen noch nicht erkennbar war. Sie ist auch gekennzeichnet durch das erstmalige Auftreten von Methionin, Phenylalanin und ,z", Substanzen, die bei den Winterfilets nicht nachweisbar waren. Nach 6 Tagen macht sich auf den Papierchromatogrammen eine noch nicht identifizierte rosagefärbte Substanz auf der Höhe von Valin (Lösungsmittel Butanol-Eisessig-Wasser $4: 1: 1$ ) bemerkbar.

Bei den tiefgefrorenen Filets werden innerhalb von 15 Tagen keine bemerkenswerten Veränderungen gefunden.

\section{Diskussion}

Nach Shewan und Jones (1957) tritt beim Dorsch zwischen dem 10. und 12. Tag der Lagerung auf Eis eine kritische Periode in der Dynamik der Mengenveränderungen einer Reihe von Substanzen auf; sensorisch wurde 
gleichzeitig schnelles Verderben und Einsetzen der Fäulnis beobachtet. Die kritische Phase ist wohl dem Beginn des Eiweißabbaues gleichzusetzen. Das Eiweißmolekül wird in seiner Gesamtheit von proteolytischen Bakterien angegriffen (MEYER 1962), so daß die dann erfolgende Zunahme einer großen Zahl von Aminosäuren, wie sie in manchen Tabellen beschrieben wird, verständlich ist. Bei Kabeljau, Seelachs und Schellfisch werden nach Bramstedt

Tabelle 11: Einsetzen der kritischen Periode der Verderbnis

\begin{tabular}{|c|c|c|c|c|}
\hline Fischart & \multicolumn{2}{|c|}{ Lagerung und Behandlung } & nach Tagen & Intensität \\
\hline Kabeljau & \multicolumn{2}{|l|}{ Filets in Eis } & später als 12 & ? \\
\hline Knurrhahn & \multirow{2}{*}{\multicolumn{2}{|c|}{$\begin{array}{l}\text { Außentemperatur } \\
\text { Eis }\end{array}$}} & $3-7$ & sehr stark \\
\hline & & & $13-20$ & mittel \\
\hline Glausauge & \multicolumn{2}{|l|}{ Außentemperatur } & $7-8$ & sehr stark \\
\hline & \multicolumn{2}{|l|}{$\begin{array}{l}\text { ganze Fische } \\
\text { Filets }\end{array}$} & $\begin{array}{l}17 \\
19\end{array}$ & $\begin{array}{l}\text { stark } \\
\text { stark }\end{array}$ \\
\hline \multirow[t]{2}{*}{ Wittling } & Außentemperatur & $\begin{array}{l}\text { ganze Fische } \\
\text { ausgen. Fische }\end{array}$ & $\begin{array}{l}3-6 \\
8-12\end{array}$ & $\begin{array}{l}\text { sehr stark } \\
\text { sehr stark }\end{array}$ \\
\hline & Eis & $\begin{array}{l}\text { ganze Fische } \\
\text { ausgen. Fische }\end{array}$ & $\begin{array}{l}17-22 \\
20-22\end{array}$ & $\begin{array}{l}\text { mittel } \\
\text { mittel }\end{array}$ \\
\hline \multirow[t]{5}{*}{ Rotbarsch } & \multicolumn{4}{|l|}{ Winter } \\
\hline & \multirow[t]{3}{*}{ Eislagerung } & ganze Fische & später als 15 & ? \\
\hline & & ausgen. Fische & später als 15 & ? \\
\hline & & Filets & später als 15 & ? \\
\hline & $\begin{array}{l}\text { Sommer } \\
\text { Eislagerung }\end{array}$ & Filets & $11-14$ & sehr stark \\
\hline
\end{tabular}

Tabelle 12: Zusammenfassung der postmortalen Konzentrationsveränderungen im Fischfleisch

\begin{tabular}{|c|c|c|c|c|c|c|}
\hline \multirow{2}{*}{ Substanz } & \multirow{2}{*}{ Knurrhahn } & \multirow{2}{*}{ Glasauge } & \multirow{2}{*}{ Wittling } & \multirow{2}{*}{ Kabeljau } & \multicolumn{2}{|c|}{ Rotbarsch } \\
\hline & & & & & Winter & Sommer \\
\hline Methionin & 0 & 0 & + & $?$ & $?$ & $+t$ \\
\hline Phenylalanin & + & + & + & $?$ & $?$ & ++ \\
\hline Tyrosin & + & + & 0 & $?$ & + & + \\
\hline Leucin/Isoleucin & $+t$ & ++ & ++ & + & + & ++ \\
\hline B-Alanin & + & + & $t$ & + & $?$ & 0 \\
\hline Histamin & + & 0 & + & $?$ & $?$ & $?$ \\
\hline Asparaginsäure & $+t$ & ++ & + & + & + & ++ \\
\hline Lysin & - & + & 0 & $?$ & $?$ & + \\
\hline$" z "$ & + & + & 十 & $?$ & $?$ & + \\
\hline${ }_{n} 13^{4}$ & + & + & + & + & + & + \\
\hline, $20^{* 6}$ & + & + & + & $?$ & $?$ & 0 \\
\hline
\end{tabular}

Bemerkungen: Die angegebenen Veränderungen einiger wichtig erscheinender Substanzen betreffen teilweise nur eine Lagerungs- oder Behandlungsart.

$++=$ starke Zunahme

$+\quad=$ geringe Zunahme oder Neuauftreten auf den Papierchromatogrammen

$0=$ keine erkennbare Veränderung

- Abnahme

? $\quad=$ noch nicht beobachtet, da kritische Phase noch nicht eingetreten

und Wurzbacher (1960) starke Veränderungen in der Konzentration einiger Aminosäuren dann gefunden, wenn das Fischfleisch nur noch den Qualitäten III bis V entspricht, d. h. wenn es sich im Sinne der Genußtauglichkeit nicht mehr um gutes oder um bereits verderbendes Fleisch handelt. Wenn wir den Zeitpunkt einer solchen kritischen Periode, den wir bei den verschiedenen hier untersuchten Arten und Lagerungsformen gefunden haben, tabellarisch zu- 
sammenfassen, dann ergibt sich das Bild der Tabelle 11. Als kritische Periode gilt dabei die Lagerungsdauer, nach welcher sich stärkere Veränderungen auf den Papierchromatogrammen bemerkbar machen. Tabelle 12 gibt eine zusammenfassende Darstellung der Veränderungen der Konzentrationen einiger wichtig erscheinender Substanzen im Verlauf der postmortalen Lagerung.

Aus diesen beiden und den früheren Tabellen sind zwei bedeutende Fakten ersichtlich:

1. Verschiedenheit des Verderbnisverlaufs bei den einzelnen Fischarten,

2. Verschiedenheit des Verderbnisverlaufs bei den verschiedenen Behandlungs- und Lagerungsarten.

Die Unterschiede sind bedingt durch zeitlich differierendes Auftreten der kritischen Phasen und durch zeitlich, qualitativ und quantitativ verschiedenes Verhalten einiger Substanzen.

Die kritische Periode tritt verständlicherweise am frühesten bei den bei Außentemperatur gelagerten Fischen auf. Bei dieser Lagerungsart machen sich die Prozesse bei Wittling und Knurrhahn früher bemerkbar als beim Glasauge. Doch zeigen die entsprechenden Tabellen, daß die Außentemperatur bei dem Versuch mit den Glasaugen etwas niedriger war, wodurch also eine Verzögerung der Verderbnis zu erklären ist. In den eisgelagerten Kabeljaufilets hat die kritische Phase vermutlich noch nicht eingesetzt, denn die bis zum 12. Tag erkennbaren Veränderungen sind nicht so intensiv wie bei den anderen Arten. Fleck ,13" wird soeben erst nachweisbar. Auch in den Winterproben des Rotbarsches ist die kritische Phase noch nicht zu erkennen, sie dürfte jedoch bei den Filets früher beginnen, da Fleck „13“ hier bereits gut nachweisbar ist und bedeutend mehr Veränderungen vorliegen. Die kritische Phase tritt jedoch in den Sommerproben des Rotbarsches stark in Erscheinung. Es sind hier also jahreszeitliche Unterschiede in der Geschwindigkeit des Ablaufs der postmortalen Prozesse erkennbar. Solche wurden auch von Castell und Triggs (1955) und E. Ranke (1960) bei den von ihnen untersuchten Arten beschrieben.

Die Befunde bei den in Eis gelagerten Proben lassen erkennen, daß die kritische Periode bei den im Winter gefangenen Fischen ziemlich gleichzeitig eintritt, lediglich beim Rotbarsch scheint sie stark verzögert zu sein. Ihre Intensität ist jedoch bei den einzelnen Arten unterschiedlich.

Die Veränderungen beginnen bei den nicht ausgenommenen Wittlingen bei Außentemperatur und auch in Eis früher als bei den ausgeweideten Tieren. Bei den ganzen Fischen treten schon nach 3-4 Tagen Deckslagerung starke Veränderungen in den Konzentrationen einer großen Reihe von Substanzen auf, was bei ausgenommenen Fischen erst nach 7 Tagen beobachtet wird (Tabelle 6). Etwas schwächer ausgeprägt ist dies bei Eislagerung. Es liegt offenbar ein Einfluß der inneren Organe vor. Dieser kann möglich sein durch Diffusion von Proteinasen aus anderen Organen in die Muskulatur (SIEBERT 1957). Merkwürdigerweise erscheinen aber Histamin und die noch nicht identifizierten Substanzen "13“ und „20" und verschwinden Oxyprolin und Trimethylaminoxyd jeweils zu denselben Zeitpunkten, einerlei, ob es sich um ausgeweidete oder um ganze Fische handelt. Das dürfte wohl bedeuten, daß bei diesen Substanzen ein Einfluß der Eingeweide nicht stattfindet. Da Trimethylaminoxyd durd Bakterien abgebaut wird (BEATty und GibBons 1936; BEATTY 1938) und Histamin vorwiegend durch Bakterientätigkeit aus Histidin entsteht (s. SHEWAN 1955), kann angenommen werden, daß dieses gleichzeitige 
Neuerscheinen bzw. Verschwinden von Substanzen auf bakterieller Tätigkeit beruht - nach RANKe (1960) werden etliche freie Aminosäuren in den ersten Lagerungstagen durch Bakterien abgebaut -, während zumindest bei einem anderen Teil der Verbindungen, bei denen es sich vorwiegend um Eiweißbausteine handelt, proteolytische Prozesse vorliegen (muskeleigene Fermente oder bakterielle Proteolyten), die bei den ganzen Fischen durch Proteinasen aus anderen Organen beschleunigt sein können. Die Bakterieneinwanderung müßte vorwiegend durch die Haut erfolgt sein, nur so ist die Gleichmäßigkeit des Auftretens oder Verschwindens der besagten Stoffe zu verstehen.

Beim Rotbarsch scheint der Einfluß der Eingeweide geringer zu sein, oder er ist in der Versuchszeit noch kaum erkennbar. Hier ist bei den ausgenommenen Fischen das anfängliche Verschwinden und spätere Wiederauftreten von Leucin/Isoleucin bemerkenswert, das auch bei Filets des Rotbarsches und des Glasauges beobachtet wird. E. Ranke und Bramstedt (1955) sowie E. Ranke (1960) berichteten über den gleichen Befund bei Filets von Seelachs und Lengfisch, wo auch Valin sich so verhielt. E. RANKE sieht darin einen Hinweis auf eine selektive Abspaltung durch Mikroorganismen. Es handelt sich hier wohl um den Verbrauch bestimmter freier Aminosäuren durch frühzeitig auftretende Mikroorganismen. Das spätere Wiederauftreten dieser Substanzen dürfte auf Eiweißspaltung zurückzuführen sein. Dieser Vorgang wurde nur bei ausgenommenen Fischen oder Filets der genannten Arten beobachtet, nicht aber bei nicht ausgeweideten Fischen. Die bisherigen Ergebnisse zeigen also, daß der Verderbnisablauf bei diesen Behandlungsarten verschieden sein kann. Das geschilderte Verhalten von Leucin und Isoleucin wurde nicht bei den Rotbarschfilets des Sommerfanges und nicht bei Wittling, Knurrhahn und Kabeljau gefunden, obwohl die Leucin-/Isoleucin-Mengen in den frischgefangenen Fischen dieser Versuchsreihe zum Teil der des Rotbarsches und des Glasauges entsprachen.

Bei sämtlichen Tiefgefrierversuchen wurden keine deutlichen Veränderungen des Bestandes der niedrigmolekularen Stickstoffverbindungen gefunden. Das Ergebnis steht in Einklang mit Arbeiten anderer Autoren.

Das Einbeziehen von Filets in das Arbeitsprogramm sollte Auskunft darüber geben, inwieweit sich der Verderbnisablauf der Filets von dem ganzer oder ausgenommener Fische unterscheidet. Ein Vergleich der Behandlungsarten ist besonders bei den im Winter gefangenen Rotbarschen möglich. Im Filet beginnen die Verderbnisvorgänge früher als bei den anderen beiden Behandlungsarten. Das ist ersichtlich aus der früh erkennbaren Abnahme besonders von Trimethylaminoxyd, an der beginnenden Zunahme einiger Flekken auf den Papierchromatogrammen und vor allem am Auftreten der Substanz „13“, deren Bedeutung weiter unten diskutiert werden soll. Bei ausgenommenen und nicht ausgenommenen Fischen waren innerhalb der Versuchszeit nur wenige bemerkenswerte Veränderungen im Aminosäurenmuster zu erkennen. Daß hingegen bei den Filets frühere Verderbnis einsetzte, kann auf das Fehlen einer Schutzeinrichtung zurückgeführt werden. Nach verschiedenen Autoren dürfte die Haut des toten Fisches ein Angriffszentrum für Bakterien sein, von der aus sie in die Muskulatur infiltrieren und die Verderbnis fördern (vgl. Reay und Shewan 1942). Dies ist ja auch beim Wittling angenommen worden. Beim ausgenommenen Kabeljau soll hingegen nach DYER et al. (1946) die Haut mindestens 10 Tage lang ein Hindernis für Bakterien sein. Es ist ja bekannt, daß die Festigkeit der Haut der verschiedenen Fischarten sehr unter- 
schiedlich ist. So war die Haut der verderbenden Wittlinge, Glasaugen und Knurrhähne viel eher und stärker angegriffen als die von Kabeljau und Rotbarsch. Die schnelle Verderblichkeit der eisgelagerten, enthäuteten Rotbarschfilets mag daher auf das Fehlen der Haut zurückzuführen sein, die Verhältnisse wären also denen von DYER et al. beim Kabeljau beschriebenen ähnlich. Andererseits kann aber auch der Filettierprozeß den Angriff von Mikroorganismen begünstigen, da das Filettieren, besonders von Hand, wie es hier der Fall war, eine Infiltration der im lebenden Zustand keimfreien Muskulatur (Reay und Shewan 1942, Dyer et al. 1950) ermöglicht.

Es gibt aber auch Fische, deren Filets in geringerem Maße der Verderbnis ausgesetzt sind als die ganzen Fische. Dies wurde beim Glasauge beobachtet (Tabelle 5). Hier tritt der größte Teil der postmortalen Veränderungen bei den nicht ausgenommenen Fischen früher ein als beim Filet. Die Filets waren allerdings noch behäutet. Ob hier eine Schutzfunktion der Haut in der ersten Phase angenommen werden kann, ist zweifelhaft, da die Haut des Glasauges sehr schnell schleimig und deformiert wird. Daß die Verderblichkeit der ganzen Fische aber größer war als die der Filets, könnte durch sehr starke Beeinflussung der Muskulatur der ganzen Fische durch innere Organe (Proteinasen, Bakterien) bedingt sein. Möglicherweise spielt hierbei auch die Festigkeit des Gewebes eine Rolle. Im letzten Untersuchungsstadium ist der Unterschied zwischen ganzen Fischen und Filets des Glasauges nicht mehr so groß.

Von allen untersuchten Substanzen, deren Konzentration sich im Lauf der Verderbnis in bemerkenswerter Weise verändert oder welche erst in einem späteren Stadium auftreten, dürfte neben Trimethylaminoxyd die noch nicht identifizierte Verbindung „13“ am auffälligsten sein. Eine Abnahme von Trimethylaminoxyd wurde bei den Fischen, bei denen die kritische Phase in der Versuchszeit auftrat, immer gefunden. Es herrscht also Übereinstimmung mit den Ergebnissen zahlreicher Forscher. Der Fleck „13“ tritt auf den Papierchromatogrammen aller Lagerungs- und Behandlungsarten des Knurrhahns, Wittlings, Glasauges und Kabeljaus sowie in den Filets des Rotbarsches auf. Diese Substanz erscheint jeweils kurz vor oder während der kritischen Phase und dürfte daher als Indikator des beginnenden Verderbens von größerer Bedeutung sein als die übrigen Verbindungen. Ob „13“ mit einer der unbekannten Substanzen von SHewan (1955) oder Hughes (1959) identisch ist, kann wegen verschiedenartiger Methodik noch nicht geklärt werden. Das Auffinden der Substanz mit der hier angewandten Methode ist nur bei zweimaligem Lauf in den beiden Lösungsmittelgemischen möglich. Der Fleck deckt sich auf den Papierchromatogrammen fast mit Threonin und Oxyprolin, ist aber von diesen Aminosäuren auf Grund anderer Anfärbbarkeit mit Ninhydrin, Folins Reagens und Chloramin gut zu unterscheiden. Fleck „13“ wurde unter 45 frischgefangenen Fischarten nur bei Seeteufel, Seehase, grauem Knurrhahn, Nagelrochen und Dornhai gefunden (Schaefer 1962 a, b). Daß „13“ nur bei den Filets des Rotbarsches, nicht aber bei den anderen Behandlungsarten gefunden wurde, liegt wohl daran, daß bei diesen das entsprechende Verderbnisstadium noch nicht erreicht war, worauf schon mehrfach hingewiesen wurde.

Das Auftreten von Histamin im verderbenden Fisch steht nach Shewan (1955) in Zusammenhang mit dem Vorkommen von freiem Histidin in der Muskulatur frischgefangener Fische. Bei solchen Arten, die eine große Menge freies Histidin enthalten, soll daher der Histamintest auf den Verderbniszustand positiv ausfallen. So wurde Histamin auch bei verdorbenen histidin- 
reichen Fischen wie Hering (Hughes 1959), Makrele (Srmmd und Hrbrki 1954, Geiger 1944) und Sardine (Geiger) gefunden. Shewan (1955) beobachtete das Auftreten von Histamin auch bei eisgelagerten Makrelen nach 8tägiger Lagerung. In der vorliegenden Untersuchung wurde Histamin lediglich beim Knurrhahn und bei den bei Außentemperaturen gelagerten Wittlingen nachgewiesen. Es ist bemerkenswert, daß bei diesen Arten freies Histidin nur in sehr geringer Menge im frischen Fisch vorlag (Schaefer 1962 a). Das Auftreten von Histamin ist also nicht immer mit dem Vorkommen großer Initialmengen an freiem Histidin verknüpft. Bei den übrigen Arten wurde Histamin nicht gefunden, obwohl zumindest beim Glasauge und bei den Rotbarschfilets des Sommerfanges das betreffende Verderbnisstadium vorgelegen haben dürfte.

Nach 14tägiger Lagerung bei $+4^{0}$ bis $+7^{0}$ erscheint beim Seelachs und Lengfisch ein unbekannter Fleck " $z^{*}$ auf den Papierchromatogrammen (E. Ranke 1960). Die Konzentration dieser Substanz nimmt mit fortschreitender Lagerung zu. Die Autorin sieht in dem Auftreten von "z" ein Kennzeichen der fortgeschrittenen Verderbnis. Dieses vermutliche Peptid (B. Ranke 1959) wurde auch bei einer größeren Zahl von Arten sofort nach dem Fang gefunden (B. Ranke 1959, Schaefer 1962 a, b). Ein stärkeres Auftreten ist, wie die hier vorgetragenen Ergebnisse zeigen, auch beim Knurrhahn im Verlaufe der Deckslagerung und beim Glasauge sowohl bei Lagerung bei Außentemperatur als auch auf Eis festzustellen. Eine geringere Zunahme der Mengen von " $\mathrm{z}^{\text {" }}$ wird beim Wittling und in den Filets der im Sommer gefangenen Rotbarsche nachgewiesen, nicht aber (oder aber erst zu einem späteren Zeitpunkt des Verderbens) bei den im Winter gefangenen Rotbarschen sowie beim Kabeljau. Auch diese Verbindung dürfte von großem Interesse für das Studium der postmortalen Veränderungen im Fischfleisch sein.

Bei fortschreitendem Verderben von Seelachs und Lengfisch nehmen die Konzentrationen von Leucin, Isoleucin, Valin und Phenylalanin zu (E. Ranke und Bramstedt 1955, E. Ranke 1960). Dasselbe gilt für den Hering (Hughes 1959, Phenylalanin wurde von ihm nicht untersucht) und Kabeljau (DiETrich 1956. Shewan und Jones 1957). Beim Schellfisch wurde jedoch eine Abnahme der Leucin- und Isoleucinmengen beobachtet, die Konzentrationen dieser Aminosäuren stiegen bei der Qualitätsstufe IV (Anfangsstadium der Verderbnis) nur unwesentlich an (Bramstedt und Wurzbacher 1960). Dieselben Autoren fanden die Höchstmengen von Leucin und Isoleucin beim Kabeljau in der Qualitätsstufe III, bei einsetzendem Verderben aber nahmen die Mengen wieder $a b$. Eine nur geringfügige Veränderung der Leucinmengen stellte Shewan (1955) bei der Makrele nach achttägiger Lagerung fest, bei der die Fische schon starke Verderbnisanzeichen aufwiesen, erkennbar am Auftreten von Histamin. Bei den von mir untersuchten Fischarten ist eine starke $\mathrm{Zu}-$ nahme von Leucin und Isoleucin bei Knurrhahn, Glasauge, Wittling (bei Eislagerung in geringerem Umfang) und Rotbarsch (Sommerfilets) festzustellen. Auch beim Kabeljau tritt nach 9-12 Tagen Lagerung auf Eis Leucin/ Isoleucin etwas verstärkt auf. Eine Qualitätsprüfung konnte nicht durchgeführt werden. Das Aminosäurenmuster nach dieser Lagerungszeit zeigt, daß noch keine wesentliche Verderbnis eingetreten ist, es kann sich also um Qualität III handeln.

Phenylalanin verhält sich nach E. Ranke (1960) wie auch nach den eigenen Befunden gleichsinnig wie Leucin und Isoleucin. 
Während bei Kabeljau, Seelachs und Schellfisch eine Zunahme von Lysin während der Lagerung erfolgt (Jones 1954, Bramstedt 1957, Shewan und Jones 1957; E. Ranke 1960), nimmt die Konzentration dieser Aminosäure beim Hering ab (Hughes 1959). Das unterschiedliche Verhalten dieser Aminosäure bei verschiedenen Fischarten zeigen auch die jetzigen Ergebnisse.

Die unbekannte Substanz „20", die nach Meyer (persönliche Mitteilung) mit Cadaverin identisch ist, wurde nur bei den bei Außentemperatur gelagerten Fischen gefunden. Wahrscheinlich war hier ein stärkerer Verderbnisgrad erreicht als bei den anderen Versuchen. Allerdings konnte papierchromatographisch noch keine deutliche Abnahme von Lysin erkannt werden.

Ein Studium der Tabellen läßt erkennen, daß eine Reihe weiterer Substanzen im Verlauf der Lagerung zu- oder abnimmt. Jedoch sind, in Übereinstimmung mit den Befunden früherer Autoren, Unterschiede von Art zu Art zu finden (vgl. Tabelle 12), so daß diese Verbindungen zur Aufstellung eines für viele Fischarten gültigen Testverfahrens der Verderbnis nicht in Frage kommen. Die einzelnen Substanzen sollen daher nicht weiter diskutiert werden. Aufzuzeigen, inwieweit Autolyse, Proteinasentätigkeit der inneren Organe oder Bakterienaktivität mit dem Verderben des Fischfleisches zusammenhängen, ist nicht $Z$ wedk dieser Arbeit. Eine klare Übersicht über den anteilmäßigen Einfluß von Mikroorganismen und muskeleigenen Fermenten brachte MEYER (1962). Eine gleichzeitige bakteriologische Untersuchung des vorliegenden Materials wäre zweifelsohne sehr fruchtbar gewesen, ließ sich aber nicht ermöglichen.

Es bleibt offen, wie die Abnahme der Mengen etlicher Substanzen in der Lagerungszeit zu erklären ist. Bei einigen Verbindungen kann es sich um bakterielle oder enzymatische Einwirkung handeln, bei anderen kann aber auch ein Auslaugen durch das Schmelzwasser des Eises bei den Eislagerungsversuchen stattgefunden haben, wie es von mehreren Autoren (z.B. Partmann 1951, Jones 1954, 1955, Shewan und Jones 1957) beschrieben worden ist. Darauf weist auch die besonders bei eisgelagerten Filets beobachtete Abnahme von Substanzen hin.

$\mathrm{Ob}$ manche Verschiedenheiten in den Konzentrationsveränderungen der Substanzen bei den unterschiedlichen Fischarten und Lagerungs- und Behandlungsweisen auf das zufällige Vorhandensein oder Fehlen der in Frage kommenden Bakterienflora beruhen, mag dahingestellt sein.

Die vorliegenden Untersuchungen zeigen, daß die postmortalen Veränderungen des Bestandes der niedrigmolekularen stickstoffhaltigen Verbindungen in der Muskulatur verschiedener Fischarten zum Teil sehr unterschiedlich verlaufen. Es sind auch Unterschiede zwischen den Lagerungsarten vorhanden. Es hat sich auch deutlich herausgestellt, wie eigentlich zu erwarten war und sich empirisch erwiesen hat, daß die Behandlungsart von großem Einfluß auf die Veränderungen während der Lagerung ist auf Grund von Einwirkungen; die von den inneren Organen oder von der Haut ausgehen. Daß bei derselben Art auch jahreszeitliche Unterschiede im Verderbnisverlauf sichtbar sind, darauf wurde ebenfalls hingewiesen. 


\section{Zusammenfassung}

Die postmortalen Veränderungen des Bestandes niedermolekularer Stickstoff verbindungen in alkoholischen Muskelextrakten von 5 frischgefangenen Seefischarten unter verschiedenen Lagerungsbedingungen wurden bei fast täglicher Probenentnahme papierchromatographisch untersucht.

$\begin{array}{ll}\text { Gadus morhua: } & \text { Filets, tiefgefroren } \\ & \text { Filets, Eislagerung } \\ \text { Trigla cuculus: } & \text { ganze Fische, Lagerung bei Außentemperatur } \\ & \text { ganze Fische, Eislagerung } \\ \text { Argentina silus: } & \text { ganze Fische, tiefgefroren } \\ & \text { ganze Fische, Lagerung bei Außentemperatur } \\ & \text { ganze Fische, Eislagerung } \\ & \text { Filets, Eislagerung } \\ \text { ganze Fische, Lagerung bei Außentemperatur } \\ \text { Merlangizs merlangus: } & \text { gangenommene Fische, Lagerung bei Außentemperatur } \\ & \text { ausgenommene Fische, Eislagerung } \\ \text { Sebastes marinus: } & \text { Filets, tiefgefroren } \\ \text { Sommerfang } & \text { Filets, Eislagerung } \\ \text { Winterfang } & \text { Filets, tiefgefroren } \\ & \text { ganze Fische, Eislagerung } \\ & \text { ausgenommene Fische, Eislagerung } \\ & \text { Filets, Eislagerung }\end{array}$

Keine nennenswerte Veränderung ist während der 2-3wöchigen Lagerungszeit bei den tiefgefrorenen Fischen $\left(-22^{\circ}\right.$ bis $\left.-26^{\circ}\right)$ erkennbar.

Starke postmortale Veränderungen treten nach wenigen Tagen bei den bei Außentemperatur $\left(3^{0}\right.$ bis $\left.12^{0}\right)$ gelagerten Fischen auf, sie sind aber bei Eislagerung verzögert.

Nicht ausgeweidete (ganze) Fische zeigen früher Verderbniserscheinungen als ausgenommene Fische. Es handelt sich hier vermutlich um eine Einwirkung von inneren Organen.

In enthäuteten Filets des Rotbarsches werden die ersten Abbauprozesse früher, in den behäuteten Filets des Glasauges später beobachtet als in ganzen oder ausgenommenen Fischen. Die Schutzwirkung der Haut und ein eventueller Einfluß des Filettierens werden diskutiert.

Der Ablauf der postmortalen Prozesse ist bei den einzelnen Arten und Lagerungsbedingungen unterschiedlich. Rotbarschfilets zeigten jahreszeitliche Unterschiede.

Die Konzentrationsveränderungen einer Reihe von Substanzen werden ausführlicher besprochen. Bei allen Versuchen, in denen die kritische Phase der Verderbnis nachgewiesen wurde, kann neben der Abnahme von Trimethylaminoxyd das Auftreten einer noch unbekannten Substanz beobachtet werden.

\section{Summary}

The post mortem changes in the pattern of nitrogenous substances in alcoholic muscle extracts from 5 freshly caught oceanic fish species stored under different conditions have been examined by means of paper chromatography.

$\begin{array}{ll}\text { Gadus morhua: } & \text { fillets, deep frozen } \\ & \text { fillets, stored in ice } \\ \text { Trigla cuculus: } & \text { ungutted, stored at open air temperatures } \\ & \text { ungutted, stored in ice }\end{array}$


Argentina silus:

Merlangius merlangus:

Sebastes marinus: summer catch winter catch ungutted, deep frozen ungutted, stored at open air temperatures ungutted, stored in ice fillets, stored in ice ungutted, stored at open air temperatures gutted, stored at open air temperatures ungutted, stored in ice gutted stored in ice fillets, deep frozen fillets, stored in ice fllets, deep frozen ungutted, stored in ice gutted stored in ice fillets, stored in ice

In fishes stored for 2 to 3 weeks in a deep freezer at temperatures of $-22^{0}$ to $-26^{0}$, no significant changes were observed. Storage at open air temperatures of $3^{0}$ to $12^{0}$ led to considerable post mortem changes beginning after a few days. Such changes are retarded if the fish are stored in ice cubes.

Spoilage of ungutted fishes began earlier than spoilage of gutted ones.

Compared to gutted or ungutted whole fishes, the first stages of spoilage appear in skinless redfish fillets earlier, but in Argentina fillets with skin later. The role of the skin as a barrier to bacterial attack and the influence of filletting were discussed.

The progression of the post mortem changes was different in the various fish species and storage conditions. Seasonal differences are observed in the redfish fillets.

The changes in concentration of several substances were discussed. Decrease of trimethylamine oxide was observed in all experiments and an unknown substance detected. It occured in all the experiments where the "critical period in the dynamics of the changes" (Shewan and Jones 1957) could be determined. The unknown substance might be expected to be of great interest in the analysis of the processes involved in the spoilage of fish muscles.

Die Arbeit wurde in der Fischereibiologischen Abteilung des Instituts für Meereskunde der Universität Kiel begonnen und in der Biologischen Anstalt Helgoland zu Ende geführt. Den Herren Institutsdirektoren danke ich herzlich für die Überlassung eines Arbeitsplatzes. Besonderer Dank gebührt den Herren Prof. Dr. Bückmann, Hamburg, Prof. Dr. KäNDler, Kiel, Dr. Aurich, List, und Dr. Meyer, Bremerhaven, für vielfache Unterstützung.

\section{Literaturverzeichnis}

B c a t ty, S. A., 1938: Studies of fish spoilage. II. The origin of trimethylamine produced during the spoilage of cod muscle press juice. J. Fish. Res. Bd. Canada, 4, 63-68.

- and Gibb on s, N.E., 1937: The measurement of spoilage in fish. J. Fish. Res. Bd. Canada, $3,77-91$.

B r a m s te d t, F, 1957: Geschmadks- und Geruchsstoffe im Fischfleisch. Arch. Fischereiwiss., 8,94-103.

- und Wurzbacher, I., 1960: Biochemische Untersuchungen zur Frage der Aufstellung von Güteklassen bei Seefischen. Fette, Seifen, Anstrichmittel, 62, 513-517.

Caste11, C. H., and Triggs, R. E., 1955: Spoilage of haddock in the trawlers at sea: the measurement of spoilage and standards of quality. J. Fish. Res. Bd. Canada, 12, 329341.

Di et rich, R., 1956: Untersuchungen über den Proteinabbau beim Kabeljau und Rotbarsch. Veröff. Inst. f. Meeresforschung in Bremerhaven, 4, 54-66. 
Dy e r, W. J., D y e r, F. E., and S now, M., 1946: Amines in fish muscle. III. Spoilage of iced eviscerated cod. J. Fish. Res. Bd, Canada, 6, 403-413.

-- and Dyer, F. E., 1950: Amines in fish muscle. IV. Spoilage in freshly cut cod fillets. J. Fish. Res. Bd. Canada, 7, 580-584.

Geiger, E., 1944: Histamine content of unprocessed and canned fish. A tentative method for quantitative determination of spoilage. Food Res., 9, 293.

Hugh es, R. B., 1959: Chemical studies on the herring (Clupea harengus). II. The free amino-acids of herring flesh and their behaviour during post-mortem spoilage. J. Sci. Food. Agric. 10, 558-564.

Jo nes, N. R., 1954: Factors affecting the free amino-acids composition of fresh and iced skeletal muscle of North Sea codling (Gadus callarias). Biochem. J. 58, XLVIII.

- 1955: The free amino-acids of fish. I. Taurine in the skeletal muscle of codling (Gadus callarias). J. Sci. Food. Agric., 6, 3-9.

Me y e r, V., 1962: Veränderungen des Eiweißes in steril gewonnenem Fischmuskel durch die Einwirkung von Mikroorganismen-Reinkulturen. Veröff. Inst. f. Meeresforschung in Bremerhaven. Im Drudk.

Partmann, W., 1951: Zur Frage der Bestimmung des Frischezustandes von Fischen. Z. f. Lebensmittelunters. u. -forschg. 93, 341-356.

Ranke, B., 1955: Über papierchromatographische Untersuchungen des freien und eiweißgebundenen Aminosäurenbestandes bei Krebsen und Fischen. Arch. Fischereiwiss. 6, 109 bis 113.

- 1959: Ober die nicht-eiweißgebundenen und eiweißgebundenen Aminosäurenbestände von Fischen, Mollusken und Krebsen. Arch. Fischereiwiss. 10, 117-159.

R a n ke, E., 1960: Der Einfluß der Lagerung auf die freien Aminosäuren und Peptide beim Lengfish Molva vulgaris und Seelachs Gadus virens. Arch. Fischereiwiss. 11, 18-47.

- und B ramstedt, F, 1955: Der Einfluß der Lagerungsdauer des Fischfleisches auf den Gehalt an freien Aminosäuren. Arch. Fischereiwiss. 6, 193-198.

Reay, G. A., and Shewan, J. M., 1942: The spoilage of fish and its preservation by chilling. Advanc. Food. Res, 2, 343-398.

Sch a ef er, H., 1959: Úber die Variabilität des Grundmusters freier Aminosäuren in der Rumpfmuskulatur des Rotbarsches, Sebastes marinus L., Dtsch. Hydrogr. Z., Erg. Heft, Reihe B, Nr. 3, 103-107.

- 1962 a: Freie Aminosäuren und verwandte Verbindungen in der Rumpfmuskulatur frischgefangener mariner Knochenfische. Helgol. Wiss. Meeresunters., 8, 257-275.

- 1962 b: Freie Aminosäuren und verwandte Verbindungen in der Muskulatur der Chimäre sowie einiger Elasmobranchier und Evertebraten. Helgol. Wiss. Meeresunters. 8, 280-286.

Sh ew an, J. M., 1955: The nitrogenous extractives from fresh fish muscle. III. Comparison of several flatfishes and members of the herring-madkerel-group. J. Sci. Food Agric., 6. $99-104$

- and Jo n e s, N. R., 1957: Chemical changes occurring in cod muscle during chill storage and their possible use as objective indices of quality. J. Sci. Food Agric, 8, 491-498.

$\mathrm{S}$ i e b e r t, G., 1957: Untersuchung proteolytischer Fermente in lebendfrischen Fischen. Arch. Fischereiwiss. 8, 1. Beiheft, 43-52.

$\mathrm{Simidu}, \mathrm{W}$, and $\mathrm{Hibiki}, \mathrm{S}$, 1954: Studies on Putrefaction of aquatic products. XIII Comparison of putrefaction of different kinds of fish. Bull. Jap. Soc. Sci. Fish. 20, 298. 